José Dilermando Andrade Fil ho 2 Ana Paula Salgado Carneiro 2 Mauro Lucio Nascimento Lima 2 Rodrigo Martins Santiago 3 Marco Antônio Gama 3 Carlos Al berto Santos 3 Alda Lima Fal cão 2 Reginaldo Peçanha Brazil 2

\section{Flebotomíneos de Timóteo, Estado de Minas Gerais, Brasil (Diptera: Psychodidae)1}

\author{
Sand flies in Timóteo, Minas Gerais, Brazil \\ (Diptera: Psychodidae) ${ }^{1}$
}

1 Trabal ho realizado com auxílio do CNPq/Fiocruz. 2 Laboratório de Leishmanioses, Centro de Pesquisas René Rachou, Fundação Oswaldo Cruz. Av. Augusto de Lima 1715, C. P. 1.743, Belo Horizonte, MG 30190-002, Brasil.

3 Fundação de Ação Social de Timóteo, Rua XIX de Abril 180, Timóteo, MG 35180-000, Brasil.
Abstract Sporadic cases of tegumentary lei shmaniasis have occurred in Timóteo, Minas Gerais State, basically among the rural population. In order to study the region's sand fly population, New Jersey light traps were set in seven different nei ghborhoods. Speci mens were gathered from June through October 1994, December 1994, and January through March 1995, with a total of 3,240 hours per trap. A total of 4,396 sand flies were captured, distributed among two genera and twenty species: Brumptomyia cunhai, Brumptomyia nitzulescui, Lutzomyia (Nyssomyia) whitmani, Lutzomyia (Nyssomyia) intermedia, Lutzomyia quinquefer, Lutzomyia lenti, Lutzomyia (Pintomyia) fischeri, Lutzomyia migonei, Lutzomyia sallesi, Lutzomyia termitophila, Lutzomyia aragaoi, Lutzomyia borgmeieri, Lutzomyia (Psathyromyia) Iutziana, Lutzomyia (Sciopemyia) sordellii, Lutzomyia (Pintomyia) pessoai, Lutzomyia (Trichopygomyia) longispina, Lutzomyia misionensis, Lutzomyia (Psychodopygus) davisi, Lutzomyia lanei, Lutzomyia (Pressatia) sp. The species L. (N.) whitmani was the most frequent, with $52.12 \%$ of the total, followed by L. (N.) intermedia with $34.10 \%$, and both may be involved in transmission of tegumentary leishmaniasis in the region.

Key words Cutaneous Leishmaniasis; Sand flies; Lutzomyia; Brumptomyia; Vectors

Resumo Casos esporádicos de leishmani ose tegumentar têm ocorrido no Município de Timóteo, Minas Gerais, basi camente na população rural. Para conhecer a fauna de flebotomíneos da região, foram instaladas sete armadil has lu minosas de New Jersey na cidade, em sete diferentes bairros. As coletas foram realizadas no período de junho a outubro de 1994, dezembro de 1994 e janeiro a março de 1995, com um total de 3.240 horas por armadilha. Foram capturados 4.396 flebotomíneos, distribuídos em dois gêneros e vinte espécies: Brumptomyia cunhai, Brumptomyia nitzulescui, Lutzomyia (Nyssomyia) whitmani, Lutzomyia (Nyssomyia) intermedia, Lutzomyia quinquefer, Lutzomyia lenti, Lutzomyia (Pintomyia) fischeri, Lutzomyia migonei, Lutzomyia sallesi, Lutzomyia termitophila, Lutzomyia aragaoi, Lutzomyia borgmeieri, Lutzomyia (Psathyromyia) lutziana, Lutzomyia (Sciopemyia) sordellii, Lutzomyia (Pintomyia) pessoai, Lutzomyia (Trichopygomyia) longispina, Lutzomyia misionensis, Lutzomyia (Psychodopygus) davisi, Lutzomyia lanei, Lutzomyia (Pressatia) sp. A espécie L. (N.) whitmani foi a mais freqüente com 52,12\%, seguida de L. (N.) intermedia com 34,10\%, eambas podem estar parti cipando da transmissão deleishmaniose cutânea na região.

Palavras-chave Leishmaniose Tegumentar; Flebotomíneos; Lutzomyia; Brumptomyia; Vetores 


\section{Introdução}

Flebotomíneos são insetos bastante estudados devido a sua capacidade vetorial e ao fato de as fêmeas de algumas espécies do gênero Lutzomyia serem vetoras de protozooses, entre estas as leishmanioses, que constituem graves problemas de saúde no Brasil (Lainson, 1983). A maioria das espécies do Novo Mundo habita áreas de florestas primárias e ou secundárias, sendo estes locais os possíveis criadouros. Em função das modificações ambientais impostas pelo homem, estes insetos vêm se adaptando a ambientes rurais e periurbanos (Lima, 1986; Brazil et al., 1989).

O Município de Timóteo, localizado em Minas Gerais, na região do Vale do Aço, apresenta casos esporádicos de leishmaniose cutânea, sendo estes observados basicamente na população rural. Esta cidade é limítrofe com o Parque Estadual do Rio Doce, tendo $10 \%$ deste em seus limites.

O presente trabalho tem como objetivo conhecer a fauna de flebotomíneos em sete bairros de Timóteo, utilizando para isso armadilha luminosa de New Jersey.

\section{Material e métodos}

Os insetos foram obtidos através de coletas em sete bairros da cidade, utilizando-se armadilha fotossensível de New Jersey, uma em cada bairro, assim distribuídas: São José - armadilha 1; Macuco - armadilha 2; Limoeiro - armadilha 3; Primavera - armadilha 4; Novo Horizonte - armadilha 5; Funcionários - armadilha 6; Alvorada - armadilha 7.

As armadilhas 2 e 5 estavam próximas à área de mata do Parque Estadual do Rio Doce, que se localiza a 205 quilômetros de Belo Horizonte e apresenta uma área de 35.974 hectares com florestas tropicais primárias, que, após a década de 60 , sofreu algumas modificações, com introdução de matas secundárias. Sua altitude varia entre 230 e 515 metros do nível do mar.

As coletas foram realizadas nos períodos de junho a outubro de 1994, dezembro deste mesmo ano e janeiro a março de 1995, totalizando 3.240 horas de exposição para cada armadilha. Os insetos foram recolhidos e triados semanalmente, fixados em álcool a 70\% e enviados ao Laboratório de Leishmanioses do Centro de Pesquisas René Rachou, Fiocruz, onde foram processados e montados em líquido de Berlese, entre lâmina e lamínula. A identificação seguiu a classificação proposta por Young \& Duncan (1994).

\section{Resultados e discussão}

A armadilha de New Jersey, apesar de ser utilizada para monitoramento de dipteros em zonas urbanizadas, e não ser específica para flebotomíneos, obteve bons resultados, possivelmente por causa da alta inci dência de flebotomíneos na região. Um total de 4.396 flebotomíneos foram capturados e assinalou-se a presença de vinte espécies, distribuídas em dois gêneros, Brumptomyia e Lutzomyia. As espécies identificadas foram: Brumptomyia cunhai (Mangabeira, 1942), Brumptomyia nitzulescui (Costa Lima, 1932), Lutzomyia (Nyssomyia) whitmani (Antunes \& Coutinho, 1939), Lutzomyia (Nyssomyia) intermedia (Lutz \& Neiva, 1912), Lutzomyia quinquefer (Dyar, 1929), Lutzomyia lenti (Mangabeira, 1938), Lutzomyia (Pintomyia) fischeri (Pinto, 1926), Lutzomyia migonei (França, 1920), Lutzomyia sallesi (Galvão \& Coutinho, 1939), Lutzomyia termitophila Martins, Falcão \& Silva, 1964, Lutzomyia aragaoi (Costa Lima, 1932), Lutzomyia borgmeieri Martins, Falcão \& Silva, 1972, Lutzomyia (Psathyromyia) lutziana (Costa Lima, 1932) Lutzomyia (Sciopemyia) sordellii (Shannon \& Del Ponte, 1927) Lutzomyia (Pintomyia) pessoai (Coutinho \& Barreto, 1940) Lutzomyia (Trichopygomyia) Iongispina (Mangabeira, 1942) Lutzomyia misionensis (Castro, 1939), Lutzomyia (Psychodopygus) davisi (Root, 1934), Lutzomyia Ianei (Barreto \& Coutinho, 1941), Lutzomyia (Pressatia) sp.

A Tabela 1 mostra o total de flebotomíneos identificados até o nível de espécie capturados em cada bairro durante o período de estudo. Seis exemplares não foram identificados por estarem bastante danificados, e em outros doze só foi possível a identificação até o nível de subgênero, sendo determinadas como Lutzomyia (Nyssomyia) sp. Estes exemplares são próximos de L. (N.) intermedia, com algumas variações na forma da espermateca. As fêmeas de Brumptomyia não foram identificadas até o nível de espécie devido à semel hança existente entre todas as fêmeas deste gênero (Martins et al., 1978), o mesmo ocorrendo com a única fêmea coletada do subgênero Pressatia (Young \& Duncan, 1994).

A armadilha 2, localizada no bairro $\mathrm{Macu}$ co, e a armadilha 5, no bairro Novo Horizonte, apresentaram o maior número de flebotomíneos capturados (40,99\% e 42,34\%, respectivamente). Este fato se deve à proximidade destes bairros com o Parque Florestal do Rio Doce, os quais tiveram ainda uma maior variedade da fauna flebotomínica, tendo sido capturadas 14 espécies em cada um deles. As armadilhas 1 
Tabela 1

Número de exemplares e total de espécies de flebotomíneos capturados por bairros na cidade de Timóteo, Minas Gerais, no período de junho a outubro de 1994, dezembro de 1994 e janeiro a março de 1995.

\begin{tabular}{|c|c|c|c|c|c|c|c|c|}
\hline Espécies & $\begin{array}{l}\text { Arm. } 01 \\
\text { São J osé }\end{array}$ & $\begin{array}{l}\text { Arm. } 02 \\
\text { Macuco }\end{array}$ & $\begin{array}{c}\text { Arm.03 } \\
\text { Limoeiro }\end{array}$ & $\begin{array}{c}\text { Arm. } 04 \\
\text { Primavera }\end{array}$ & $\begin{array}{c}\text { Arm. } 05 \\
\text { Novo Horizonte }\end{array}$ & $\begin{array}{c}\text { Arm. } 06 \\
\text { Funcionários }\end{array}$ & $\begin{array}{l}\text { Arm. } 07 \\
\text { Alvorada }\end{array}$ & Total \\
\hline L. whitmani & 23 & 629 & 30 & 75 & 1.407 & 11 & 116 & 2.291 \\
\hline L. intermedia & 09 & 1.010 & 75 & 64 & 291 & 06 & 44 & 1.499 \\
\hline L. quinquefer & 01 & 61 & 01 & 66 & 76 & 00 & 31 & 236 \\
\hline L. lenti & 08 & 21 & 26 & 31 & 02 & 01 & 25 & 114 \\
\hline L. migonei & 00 & 20 & 02 & 00 & 36 & 00 & 01 & 59 \\
\hline L. sallesi & 04 & 01 & 02 & 07 & 26 & 01 & 00 & 41 \\
\hline L. termitophila & 01 & 01 & 00 & 02 & 03 & 00 & 00 & 07 \\
\hline L. aragaoi & 00 & 00 & 01 & 00 & 03 & 02 & 00 & 06 \\
\hline L. Iutziana & 00 & 01 & 00 & 00 & 05 & 00 & 00 & 06 \\
\hline L. borgmeieri & 00 & 00 & 02 & 01 & 02 & 00 & 00 & 05 \\
\hline L. sordellii & 00 & 03 & 01 & 00 & 00 & 00 & 00 & 04 \\
\hline L. pessoai & 00 & 00 & 00 & 00 & 00 & 01 & 00 & 01 \\
\hline L. longispina & 00 & 01 & 00 & 00 & 00 & 00 & 00 & 01 \\
\hline L. misionensis & 00 & 00 & 00 & 00 & 01 & 00 & 00 & 01 \\
\hline L. davisi & 00 & 01 & 00 & 00 & 00 & 00 & 00 & 01 \\
\hline L. Ianei & 00 & 01 & 00 & 00 & 00 & 00 & 00 & 01 \\
\hline L. (Pressatia) sp. & 00 & 00 & 01 & 00 & 00 & 00 & 00 & 01 \\
\hline B. nitzulescui & 00 & 03 & 01 & 01 & 09 & 00 & 00 & 14 \\
\hline B. cunhai & 00 & 01 & 00 & 01 & 01 & 00 & 00 & 03 \\
\hline Total & 46 & 1.777 & 142 & 250 & 1.898 & 23 & 224 & 4.360 \\
\hline
\end{tabular}

Arm. = armadilha.

(bairro São José) e 6 (bairro Funcionários) apresentaram o menor rendimento de exemplares capturados, 1,07\% e 0,55\%, respectivamente.

A espécie L. sallesi apareceu com mais freqüência na armadilha 5 , e as espécies $L$.(N.) whitmani, L. (N.) intemedia, L. (P.) fischeri e L. migonei foram capturadas em maior número nas armadilhas 2 e 5. Nas armadilhas 2, 4, 5 e 6, houve grande número de L. quinquefer coletados, e L. Ienti apresentou baixo rendimento na armadilha 5, sendo bastante coletada nas armadilhas 2, 3, 4 e 7 .

L. (N.) whitmani foi a espécie predominante na área de estudo. Esta espécie é incriminada como vetora (ou suspeita de transmissão) de leishmanioses em diversas regiões do país inclusive na região sudeste (Lainson et al., 1989; Tolezano et al., 1992; Queiroz et al., 1994).

A espécie L. (N.) intermedia também teve uma alta proporção de exemplares capturados. Esta espécie apresenta alta antropofilia, sendo suspeita de transmitir Leishmania no sudeste do Brasil (Rangel et al., 1984). Os exemplares desta espécie capturados na área de estudo apresentaram-se com al to polimorfismo na espermateca, tanto no número de anela- ções, quanto na forma da cabeça, como observado por Marcondes (1996), entretanto consideramos todos estes exemplares como L. (N.) intermedia, estando o material reservado para estudos posteriores.

L. (P.) fischeri é uma espécie bastante antropofílica (Gomes et al., 1989), sendo suspeita de transmitir leishmanioses em outras regiões do Brasil. Outra espécie capturada na área de estudo, L. migonei, é também antropofílica (Forattini, 1973), e diversos autores relataram a presença de infecção natural neste flebotomíneo (Pessoa \& Coutinho, 1941; Pifano, 1943). Rangel et al. (1986), em captura comparativa homem/cão, verificaram que L. migonei suga o homem em maior proporção, podendo estar participando na transmissão da leishmaniose canina.

Outras espécies que apresentaram maior freqüência na área foram L. sallesi , L. quinquefer, que se al imenta de animais de sangue frio (Deane \& Deane, 1957) e não apresenta interesse médico, e L. Ienti. Esta última foi encontrada naturalmente infectada com promastigotas na Bahia (Sherlock, 1996). Sherlock relata, ainda, a alta antropofilia da espécie na popula- 
ção deste estado. Entretanto, Brazil et al. (no prelo) estudando uma população de L. Ienti, proveniente de Betim, Minas Gerais, verificaram que a espécie não se mostrou antropofílica no campo, nem no laboratório.

Das vinte espécies por nós capturadas na área de estudo, apenas L. borgmeieri não havia sido ainda registrada para a região do vale do Aço, e este é o primeiro registro de todas elas para o Município de Timóteo (Martins et al., 1978).

\section{Referências}

BRAZIL, R. P.; BRAZIL, B. G.; GOUVEA, M. C.; DE ALMEIDA, D. L.; DE OLIVEIRA, M. S. P. \& MENEZES, J. A., 1989. Epidemiological studies on cutaneous laishmaniasis in the state of Rio de Janeiro, Brazil. Domestic and peridomestic sand fly fauna. In: Leishmaniasis: The Current Status and New Strategies for Control (D. T. Hart, ed.), pp. 159-164, New York: Plenum.

BRAZIL, R. P.; CARNEIRO, V. L.; ANDRADE FILHO, J. D.; ALVES, J. C. M. \& FALCÃO, A. L., Biology of Lutzomyia lenti (Mangabeira) (Diptera: Psychodidae). Anais da Sociedade Entomológica do Brasil, (no prelo).

DEANE, L. M. \& DEANE M. P., 1957. Observações sobre abrigos e criadouros de flebótomos no noroeste do Estado do Ceará. Revista Brasileira de Malariologia e Doenças Tropicais, 9:225-246.

FORATTINI, O. P., 1973. Entomologia Médica. Vol. 4, São Paulo: Edgard Blücher/Edusp.

GOMES, A. C.; BARATA, M. S.; ROCHA E SILVA, E. O. \& GALATI, E. A. B., 1989. Aspectos ecológicos da leishmaniose tegumentar americana. 6. Fauna flebotomínica antropofílica de matas residuais situadas na região centro-nordeste do Estado de São Paulo. Revista do Instituto de Medicina Tropical deSão Paulo, 31:32-39.

LAINSON, R., 1983. The american leishmaniasis: some observations on the their ecology and epidemiology. Transactions of the Royal Society of Tropical Medicine and Hygiene, 77:569-596.

LAINSON, R.; BRAGA, R. R.; SOUZA, A. A.; POVOA, M. M.; ISHIKAWA, A. Y. \& SILVEIRA, F. T., 1989. Leishmania (Viannia) shawi sp. n., a parasite of monkeys, sloths and procyonids in Amazonian Brazil. Annales de Parasitologie Humaine e Comparé, 64:200-207.

LIMA, L. C., 1986. Ruralização de Lutzomyia intermedia, um provável caso de pré-adaptação. Revista de Saúde Pública, 20:102-104.

MARCONDES, C. B., 1996. A redescription of Lutzomyia (Nyssomyia) intermedia (Lutz \& Neiva, 1912), and resurrection of L. neivai (Pinto, 1926) (Diptera, Psychodidae, Phlebotominae). Memórias do Instituto Oswaldo Cruz, 91:457-462.
MARTINS, A. V.; WILLIAMS, P. \& FALCÃO, A. L., 1978. American Sand Flies. Rio de Janeiro: Academia Brasileira de Ciência.

PESSOA, S. B. \& COUTINHO, J. O., 1941. Infecção natural e experimental dos flebótomos pela Leishmania braziliensis, no Estado de São Paulo. O Hospital, 20:25-35.

PIFANO, C. F., 1943. Notas sobre entomologia médica venezoelana. 1 - Flebotomos transmissores de leishmaniasis tegumentari en el valle de Yaracuy. Boletim Entomológico deVenezuela, 2:99-102.

QUEIROZ, R. G.; VASCONCELOS, I. A. B.; VASCONCELOS, A. W.; PESSOA, F. A. C.; SOUZA, R. N. \& DAVID, J. R., 1994. Cutaneous leishmaniasis in Ceara state in northeastern Brazil: incrimination of Lutzomyia whitmani (Diptera: Psychodidae) as a vector of Leishmania braziliensis in Baturite municipality. American Journal of Tropical Medicineand Hygiene, 50:693-698.

RANGEL, E. F.; SOUZA, N. A.; WERMELINGER, E. D. \& BARBOSA, A. F., 1984. Infecção natural de Lutzomyia intermedia (Lutz \& Neiva, 1912), em área endêmica de leishmaniose tegumentar americana no Estado do Rio de Janeiro. Memórias do Instituto Oswaldo Cruz, 79:395-396.

RANGEL, E. F.; SOUZA, M. A.; WERM ELINGER, E. D.; AZEVEDO, A. C. R.; BARBOSA, A. F. \& ANDRADE, C. A., 1986. Flebótomos de Vargem Grande, foco de leishmaniose tegumentar no Estado do Rio de Janeiro. Memórias do Instituto Oswaldo Cruz, 81: 347-349.

SHERLOCK, I., 1996. Ecological interactions of visceral leishmaniasis in the state of Bahia, Brazil. Memórias do Instituto Oswaldo Cruz, 91:671-683.

TOLEZANO, J. E.; NOVELLI, M. A. \& TANIGUCHI, H. $H .$, 1992. Leishmaniose tegumentar americana no Estado de São Paulo. Vı. Avaliação do comportamento antropofílico de flebotomíneos em áreas endêmicas. Memórias do Instituto Oswaldo Cruz, 87:219.

YOUNG, D. G. \& DUNCAN, M. A., 1994. Guide to the identification and geographic distribuition of Lutzomyia sand flies in México, the West Indies, Central and South America (Diptera: Psychodidae). Memoirs of the American Entomological Institute, 54. 